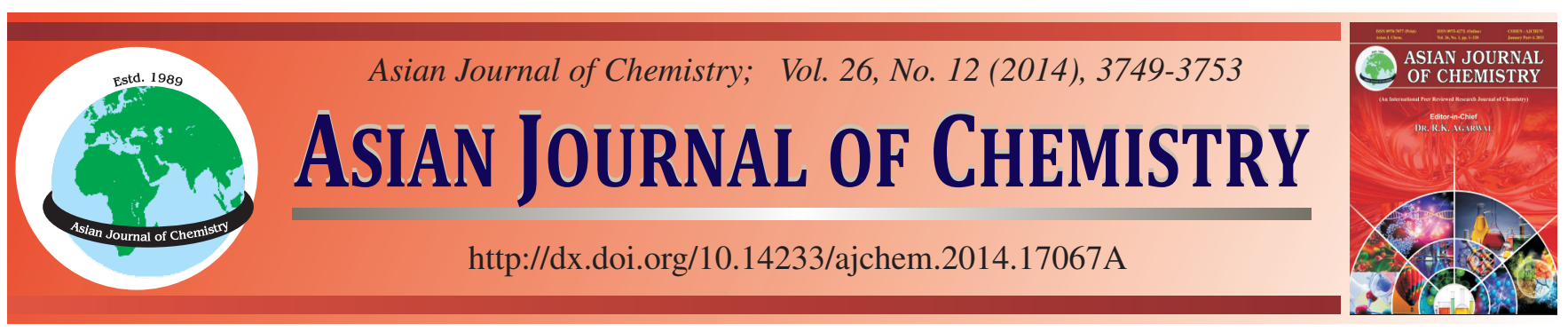

\title{
Protective Role of Arctium lappa Linn. against Arsenic Trioxide Using Silybum marianum Linn. as Standard Drug $\dagger$
}

\author{
Ann Susan Edison ${ }^{1, *}$, Uma Maheswari Krishnan ${ }^{2}$ and Brindha Pemiah ${ }^{1}$
}

${ }^{1}$ Centre for Advanced Research in Indian System of Medicine, SASTRA University, Thanjavur-613 401, India

${ }^{2}$ Centre for Nanotechnology \& Advanced Biomaterials, SASTRA University, Thanjavur-613 401, India

*Corresponding author: E-mail: umakrishnan@ sastra.edu

Published online: 5 June 2014;

AJC-15339

\begin{abstract}
Continuous exposure of humans to arsenic through long term ingestion of contaminated water and food leads to serious health problems. We have investigated the protective role of Arctium lappa extract against arsenic trioxide using Silybum marianum as standard in Wistar rats. Wistar rats were administered arsenic trioxide $(5$ and $10 \mathrm{mg} / \mathrm{kg}$ ) by oral gavage for 30 and 60 days. Results established a significant increase in certain biochemical parameters such as alkaline phosphatase, serum glutamate oxaloacetate transminase, serum glutamate pyruvate transaminase, urea and creatinine. Significant increase and decrease in haematological parameters such as lymphocytes erythrocytes were reported. Certain histopathological lesions were also seen in arsenic induced rats. Silybum marianum and Arctium lappa at a dosage of (30 and $50 \mathrm{mg} / \mathrm{kg}$ ) significantly normalized body weights, haematological and biochemical profiles in arsenic treated rats. This study demonstrates that Arctium lappa exhibits a protective role against arsenic trioxide and is as efficient as Silybum marianum.
\end{abstract}

Keywords: Arsenic trioxide, Silybum marianum, Arctium lappa, Detoxification, Biochemical, Haematology, Histopathology.

ᄂ - - - - - - - - - - - - - - - - - - - - - - - - - - -

\section{INTRODUCTION}

Arsenic is highly toxic and carcinogenic metalloid which arises from natural sources, industrial sources and from intentionally administered sources ${ }^{1}$. It exists in elemental, trivalent or pentavalent states. Arsenic trioxide is highly poisonous than arsenic pentoxide and is readily absorbed by gastrointestinal tract and on skin exposure. The permissible limit of arsenic is $10 \mathrm{ppm}$ in drinking water and above the permissible limit, arsenic trioxide causes high toxicity in humans, plants and animals ${ }^{2}$. Chronic exposure to arsenic in drinking water causes increased risk of skin, lung cancer, liver and kidney toxicity resulting in death.

At cellular level arsenic disrupts ATP production through a number of mechanisms. In citric acid cycle, it is an inhibitor of lipoic acid, a cofactor of pyruvate dehydrogenase, an enzyme involved in citric acid cycle ${ }^{1-6}$. It also disrupts oxidative phosphorylation by competing with phosphate ions involved in the process. As a result of this inhibition, there is increased production of hydrogen peroxide resulting in oxidative stress. Such metabolic interferences leads to multiple-system organ failure due to necrotic cell death ${ }^{7}$.

Mechanism of arsenic trioxide in liver: Liver forms the main target of arsenic toxicity. Chronic oral arsenic exposure leads to hepatoportal sclerosis which results in liver endothelial cell damage, which further leads to damage of parenchymal cells. High hepatic arsenic levels causes liver cirrhosis associated with hepatocellular carcinoma ${ }^{8}$. Liver fibrosis is also common in patients consuming high-arsenic contaminated food. Arsenite has a high affinity for thiol (-SH) groups in proteins and further leads to the inactivation of a variety of enzymes.

Further, it is also induces DNA hypomethylation which results in the reduction of SAM pool. This leads to the suppression of DNA methyltransferases and results in DNA methylation which further enhances genomic instability of chromosomes in mammalian cells ${ }^{9}$ (Fig. 1).

Hence, it is necessary to find out a way to eliminate these toxins from the body and to restore the normal functioning of the liver cells. The most common form of detoxification can be achieved by chelation therapy which involves the use of chelating agents to detoxify toxic substances from the body ${ }^{10}$. The commonly used chelating agents against arsenic trioxide are DMSA, penicillamine and BAL. But, these chelating agents on long time exposure causes various side effects such as nausea, vomiting, headache, elevated blood pressure and heart rate, myasthenia gravis, nephropathy, heptotoxicity, membranous glomerulonephritis and hemolysis ${ }^{11}$. 


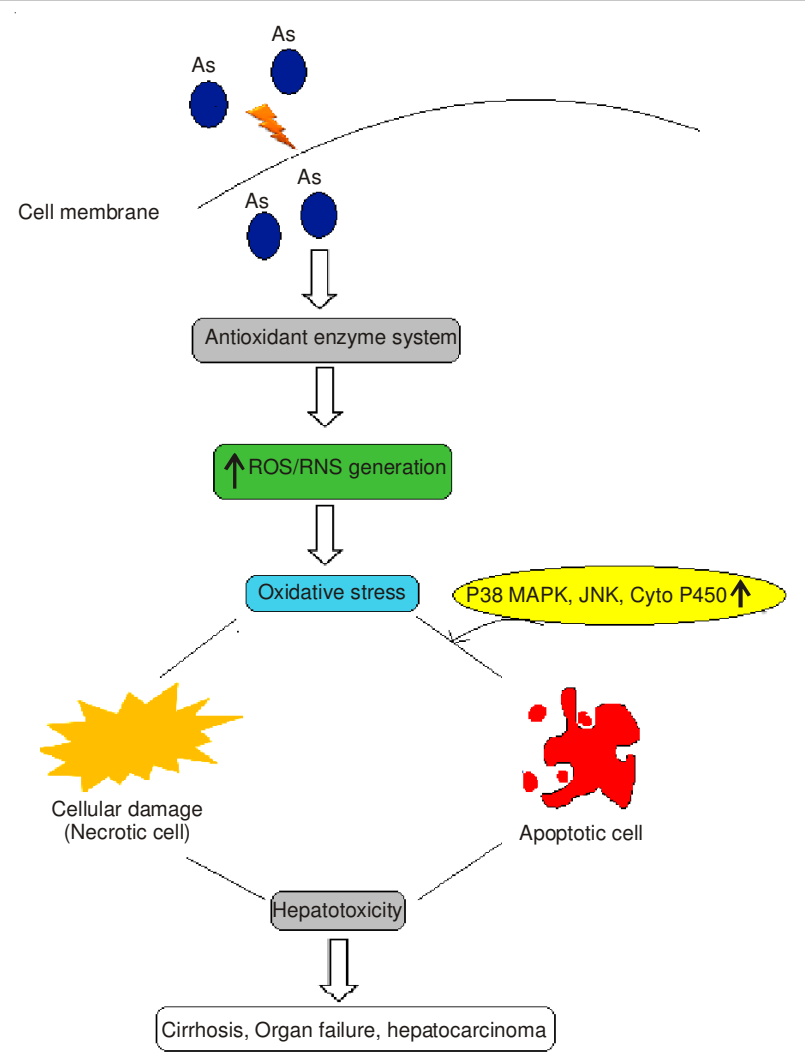

Fig. 1. Mechanisum of arsenic toxicity in liver

Whereas, indigenous drugs on the other side have shown effective and progressive relief in relation to detoxification of these metal ions without any side effects and these drugs have been used for preventive and curative aspect since long back.

S.marianum is a well known indigenous plant which is known to detoxify toxic metal ions form the blood. It has replaced the damaged cells of liver destroyed by toxins with new liver cells and proved to be more effective than vitamin $\mathrm{E}$ and $\mathrm{C}^{11}$. The active component such as silibin and silychristin plays a very important role in the detoxifying the free radicals thus preventing the oxidative stress. A. lappa commonly called as Burdock root is a good alternative as a blood purifier and possess antiinflammatory properties too ${ }^{12-16}$. The lignan arctigenin in A. lappa act as powerful antioxidants to eliminate the free radicals and proves to be as potent as standard herbal detoxifier $S$. marianum.

\section{EXPERIMENTAL}

S. marianum: The seeds of the plant were collected from Ooty, India in the month of November 2009. The seeds were identified by Dr. G.V.S. Murthy Scientific officer, Botanical Survey of India, Coimbatore. Its Accession number is BSI/ SRC/5/23/10-11TECH 409.

A. lappa: The root of the plant were collected from Simla in the month of August 2009. The seeds were identified by Dr. G.V.S Murthy Scientific officer, Botanical Survey of India, Coimbatore. Its Accession number is No. BSI/SRC/5/23/1011/TECH-1223.

All chemicals and reagents were of analytical grade. Silybum standard, $\beta$-sitosterol were purchased from Sigma chemical company (St. Louis, MO, USA).
Preparation of the plant extract: $500 \mathrm{~g}$ of dry S. marianum seeds were powdered in mechanical grinder. It was then washed with diethyl ether several times for 3 days to remove the oily content present in it. Then it was subjected to subjected to hydroalchoholic extraction.

$500 \mathrm{~g}$ of dry root were powdered, boiled in distilled water and subjected to hydroalchoholic extraction.

Animals: Wistar rats weighing 150-250 g of both the sexes were used for assessing genetic activity. Then, the animals were maintained over standard laboratory conditions and had free access to water and food. The animals were permitted to become accustomed to the environment for 7 days prior to investigational session. Then the animals were separated into different groups, each consisting of six animals which were fasted overnight prior to the experiments. Experiments on animals were performed in accordance with guidelines of the Institutional Animal Ethics Committee, SASTRA, Thanjavur.

Phytochemical screening: The presence of various phytoconstituents such as alkaloids, carbohydrates, flavanoids, glycosides, proteins, resins, tannins and steroids were analyzed qualitatively using standard protocols. GCMS and HPTLC for the sample were also carried out.

Preclinical study: Wistar rats weighing between 150$250 \mathrm{~g}$ were chosen for the study. A total of 96 Wistar rats were used to the experiment. Animals were divided into 6 in each Two time points of 30 and 60 days were fixed for the study. Arsenic trioxide of 10 and $5 \mathrm{ppm}$ were used, respectively for 30 and 60 days. Plant drugs at a dosage of $30 \mathrm{mg} / \mathrm{kg}$ and 50 $\mathrm{mg} / \mathrm{kg}$ were used as low and high doses to study their protective role in liver induced arsenic toxicity.

Sample preparation: Blood samples were collected by retroorbital method from all the animals and used in the study prior to be sacrificed for the determination of hematological and biochemical parameters. The collected blood was labeled in centrifuging tubes and allowed to clot for $0.5 \mathrm{~h}$ at normal room temperature. Then, serum was further separated by centrifugation at $3000 \mathrm{rpm}$ for $15 \mathrm{~min}$. The separated serum was used for the estimation of some biochemical parameters like Alanine aminotransferase(ALT/SGPT), Aspartate aminotransferase (AST/SGOT), Alkaline phosphatase, bilirubin, urea and creatinine. The present research had used clinical analyzer A 15 autoanalyzer, Biosystems inc., Spain to quantify the amount of enzymes.

Following sacrifice, rats were euthanized by carbon dioxide inhalation. Organs were excised and fixed in $10 \%$ neutral buffered formalin for a minimum of $48 \mathrm{~h}$, trimmed in the longitudinal plane and were processed routinely and embedded in paraffin wax. Sections were cut at 4-5 $\mu$ and stained with haematoxylin and eosin for histopathological studies.

Statistical analysis: The data are expressed as group means \pm SD. If the analysis indicated significant difference among the group means, then the group means was compared by student's $t$-test. The $p$ value of less than 0.05 was considered statistically significant.

\section{RESULTS AND DISCUSSION}

Extraction and characterization of the root of A. lappa extract: Pharmacogonostical and phytochemical characterization 
of Arctium lappa was carried out. Arctium lappa extracts were prepared using different extracting solvents. The amount of extractives obtained in each solvent was evaluated. Qualitative screening of the constituents in the extracts was also carried out. Based on the amount and nature of the extractives, the hydroethanolic extract was chosen for further trials. Gas chromatography-mass spectrometry (GC-MS) and liquid chromatography-tandem mass spectrometry (LC-MS/MS) was carried out to identify the chemical constituents in the plant. Fig. 2 shows the gas chromatography profile obtained for the hydroethanolic extract for Arctium lappa.

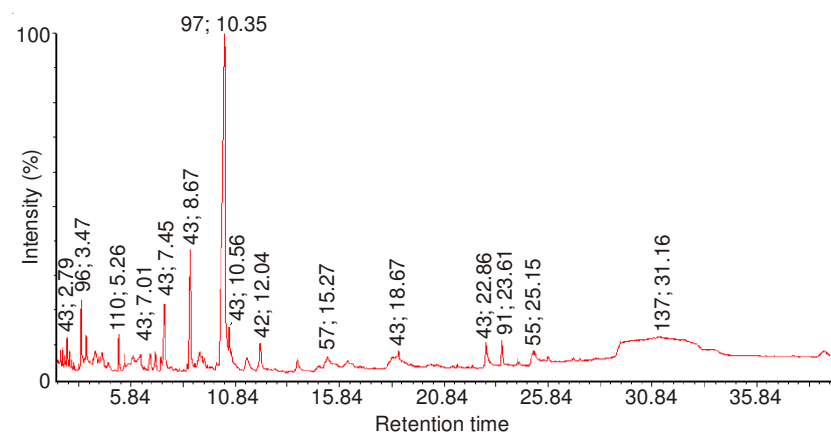

Fig. 2. GC-MS of hydroalcoholic extract of Arctium lappa

GC-MS: A total of 42 compounds belonging to the categories of esters, glycosides, lignans, palmitic and oleic acids were eluted from the column. The lignan, arctigenin, which is a key marker compound for the Arctium lappa plant was also eluted. Fig. 3 shows the fragmentation pattern of arctigenin collected after the gas chromatography of the hydroethanolic extract. The peak at the $m / z$ value of 373 corresponds to the molecular ion peak of arctigenin. The peaks at $m / z$ values of $43,55,91$, 119, 137 and 152 match with those reported earlier for the ion fragments obtained from arctigenin.

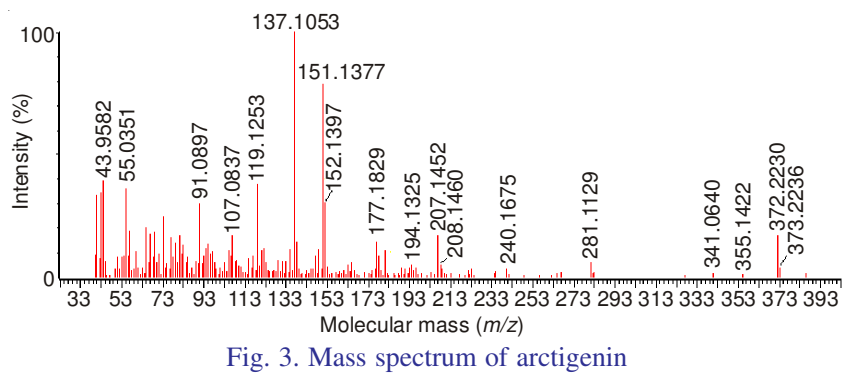

LC-MS: The hydroethanolic extract of Arctium lappa was also subjected to LC-MS/MS. The liquid chromatography was performed at a flow rate of $0.2 \mathrm{~mL} / \mathrm{min}$. The solvent system employed was a gradient of acetonitrile and acidified water (99\% water and $1 \%$ acetic acid). The gradient mobile system was commenced with $1 \%$ acetonitrile and $99 \%$ acidified water at $0.2 \mathrm{~min}$ to $75 \%$ acetonitrile at the 16th min. This was brought to $100 \%$ acetonitrile at the 19 th $\min$ and to $5 \%$ acetonitrile at $21 \mathrm{st}$ min. This condition was maintained till the end of the run at $23 \mathrm{rd} \mathrm{min}$. The UV detector was set at $325 \mathrm{~nm}$. Fig. 4 shows the mass spectrum of the compounds isolated from the liquid chromatography run. The mass spectrum was obtained using electrospray ionization and the spectrum was recorded in the negative mode. The peak at $\mathrm{m} / \mathrm{z}$ value of 372 corresponds to

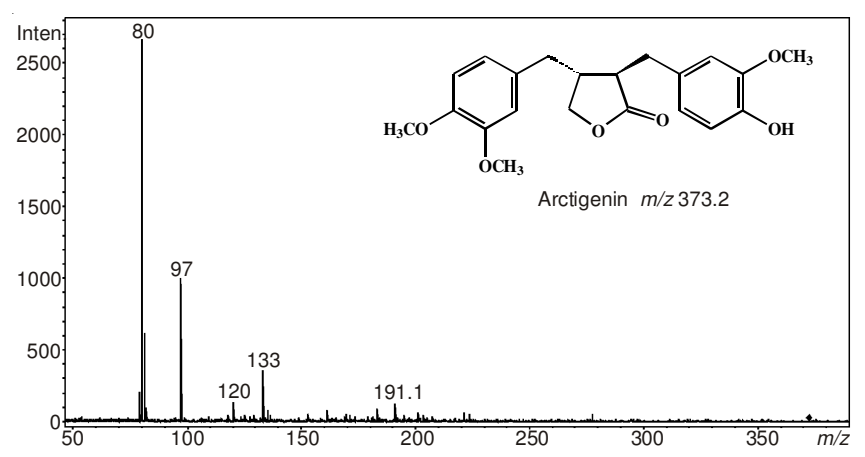

Fig. 4. LC-MS/MS profile for Arctium lappa

the M-H fragment of arctigenin. Thus both GC-MS and LCMS/MS techniques confirmed the presence of arctigenin. Other compounds such as quercetin and polyphenols were also identified in the extract. These compounds could have a major contribution to the pharmacological activities of Arctium lappa.

Experimental design: Wistar rats weighing between 120$150 \mathrm{~g}$ were selected for the study. Animals of both sexes were used in the study. Each group consisted of three male and three rats. Two time points of 30 and 60 days were fixed for the study. Arsenic toxicity was induced by administering $5 \mathrm{ppm} /$ day of arsenic trioxide $\left(\mathrm{As}_{2} \mathrm{O}_{3}\right)$ for the 60-day study and 10 $\mathrm{ppm} /$ day for the 30-day study. Plant drugs at a dosage of 30 and $50 \mathrm{mg} / \mathrm{kg}$ were used as low and high doses to study their protective role in arsenic-induced hepatotoxicity. The plant extracts were co-administered with $\mathrm{As}_{2} \mathrm{O}_{3}$ to assess their protective role. The doses were selected based on earlier reports for Silybum marianum. The following groups were used for the study:

Group I: Normal rats. Group II: Arsenic treated (10 ppm $\mathrm{As}_{2} \mathrm{O}_{3}$ for 30 days). Group III: Arsenic treated (5 ppm of $\mathrm{As}_{2} \mathrm{O}_{3}$ for 60 days). Group IV: Arctium lappa (hydroethanolic extract: $30 \mathrm{mg} / \mathrm{kg}$ ) + arsenic trioxide for 30 days. Group V: Arctium lappa (hydroethanolic extract: $50 \mathrm{mg} / \mathrm{kg}$ ) + arsenic trioxide for 30 days. Group VI: Arctium lappa (hydroethanolic extract: $30 \mathrm{mg} / \mathrm{kg}$ ) + arsenic trioxide for 60 days. Group VII: Arctium lappa (hydroethanolic extract: $50 \mathrm{mg} / \mathrm{kg}$ ) + arsenic trioxide for 60 days. Group VIII: Silybum marianum (hydroethanolic extract: $30 \mathrm{mg} / \mathrm{kg}$ ) + arsenic trioxide for 30 days. Group IX: Silybum marianum (hydroethanolic extract: $50 \mathrm{mg} / \mathrm{kg}$ ) + arsenic trioxide for 30 days. Group X: Silybum marianum (hydroethanolic extract: $30 \mathrm{mg} / \mathrm{kg}$ ) + arsenic trioxide for 60 days. Group XI: Silybum marianum (hydroethanolic extract: $50 \mathrm{mg} / \mathrm{kg}$ ) + arsenic trioxide for 60 days.

Normal group consisted of 12 animals, six of which were used for the 30-day study and the remaining was used for the 60-day study.

Biochemical parameters: The levels of alkaline phosphatase (ALP), alanine transaminase (ALT), bilirubin, urea, creatinine and aspartate transaminase (AST) were determined for the various groups and the results are presented in Fig. 5.

Toxicity of liver is commonly assessed by determination of activities of serum aminotransferases. In the group II animals, it was observed that treatment of arsenic resulted in a significant increase $(p<0.05)$ in the concentration of the enzymes ALT, AST, ALP bilirubin, urea and creatinine when compared to the group I control. The elevated levels of these 
ALP

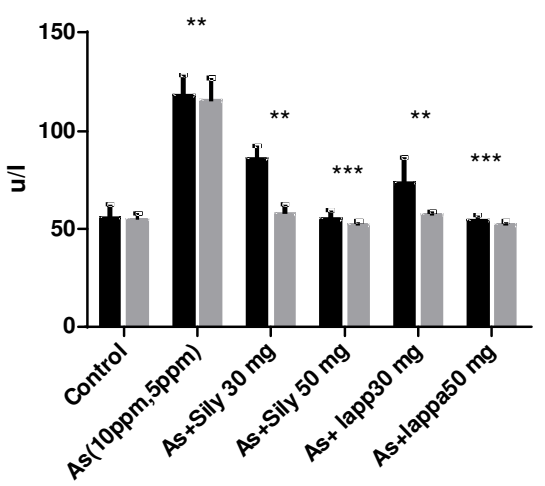

Bilirubin

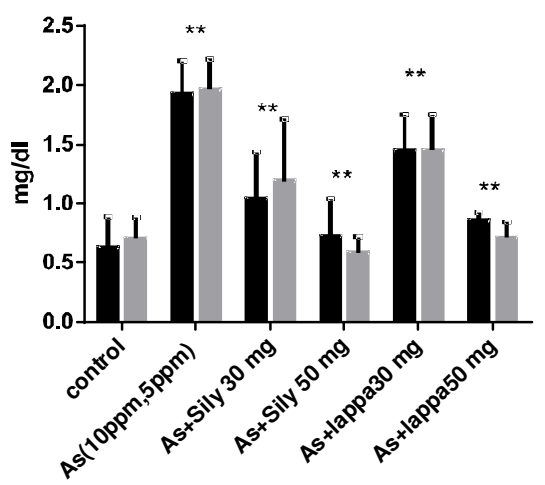

creatinine

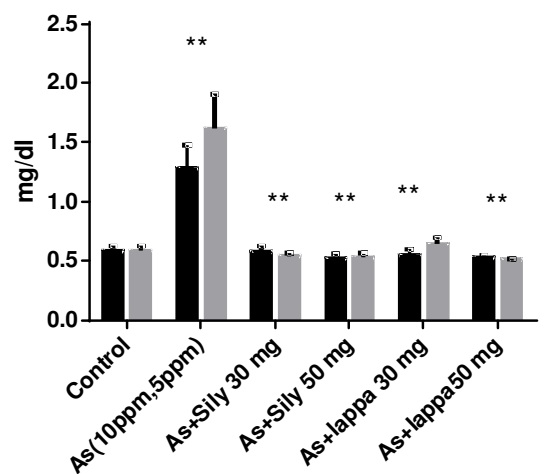

ALT

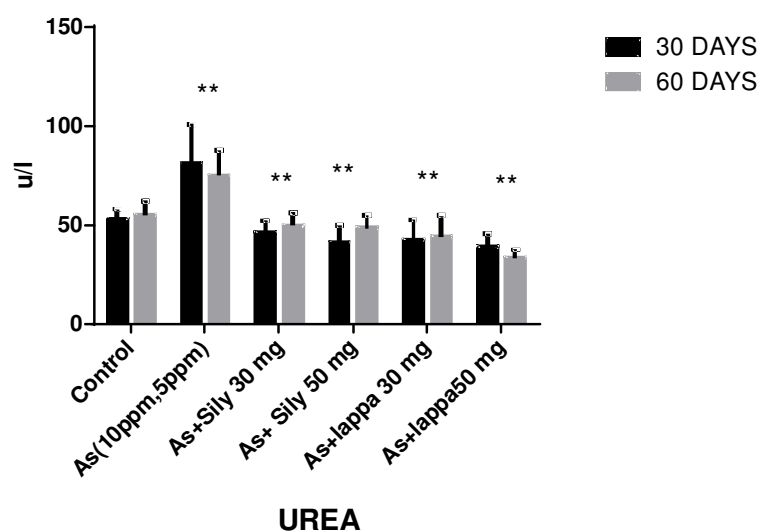

30 DAYS

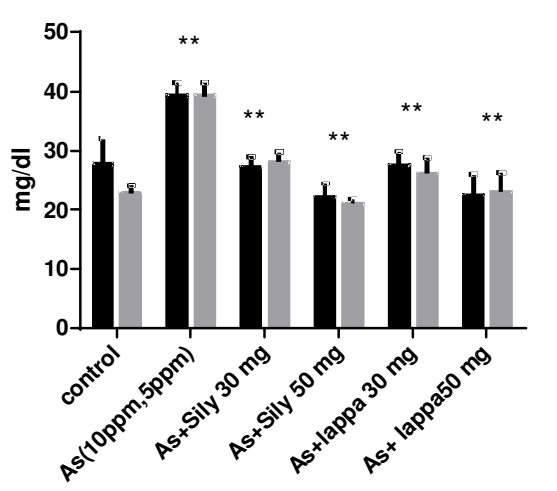

30 DAYS

60 DAYS
30 DAYS 60 DAYS

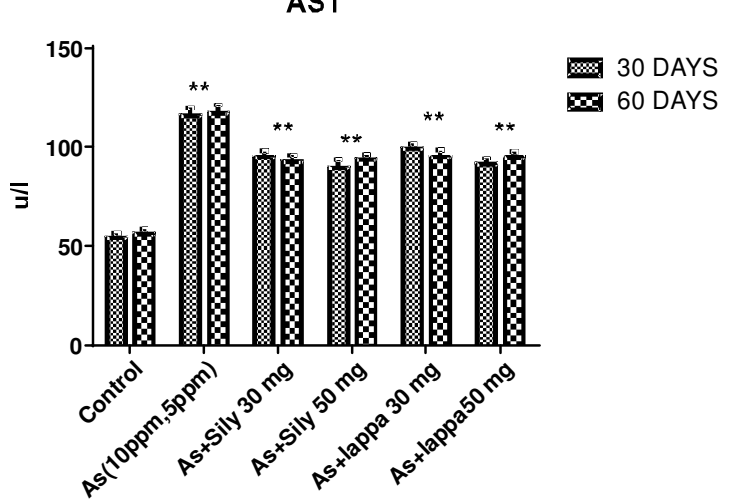

Fig. 5. Effect of various treatments on the biochemical parameters in rat blood. Data are expressed as mean \pm SD. The difference was significant $(p<0.05)$ when the drug-treated groups were compared with arsenic treated group

enzymes indicate cellular leakage and loss of functional integrity of the hepatocyte membrane on treatment with arsenic. Co-administration of rats with Silibum marianum and Arctium lappa to the rats at a dosage of 30 and $50 \mathrm{mg} / \mathrm{kg}$ restored the serum levels of ALT, AST, ALP, bilirubin, urea and creatinine towards the normal. All values except AST were found to return to normal when the plant extracts were coadministered with arsenic. The AST levels were significantly lowered when compared with the arsenic treated group. Both Arctium lappa and Silibum marinum were found to exhibit the same level of potency indicating that Arctium lappa can be effective in ameliorating the toxicity of arsenic. The coadministration resulted in protection of the hepatocytes from the detrimental effects of arsenic.

Haematological parameters: Fig. 6 shows the influence of arsenic treatment and co-administration of the plant extracts on the RBC (red blood cell) and lymphocyte counts. It was observed that treatment with arsenic resulted in a decline in the RBC count due to the inhibition of the haem porphyrin synthesis. Co-administration of Silibum marinum and Arctium lappa increased the RBC levels closer to the normal. Interestingly, while co-administration of Silibum marinum resulted in an increase in RBC levels that is slightly lower than the normal, Arctium lappa is able to restore the RBC levels to normal. This indicates that Arctium lappa may have a better positive influence in protecting the RBCs from the adverse effects of arsenic. An increase in the level of lymphocytes is noted on administration of arsenic. Co-administration with Silibum marinum and Arctium lappa resulted in restoration of the lymphocyte levels to normal. This may be attributed to the antioxidants and antiinflammatory constituents present in both plant extracts.

Histopathology: It was observed that there was no significant pathological changes in arsenic treated group when compared with the control and Silybum marianum coadministered groups. No other changes in the morphology of 

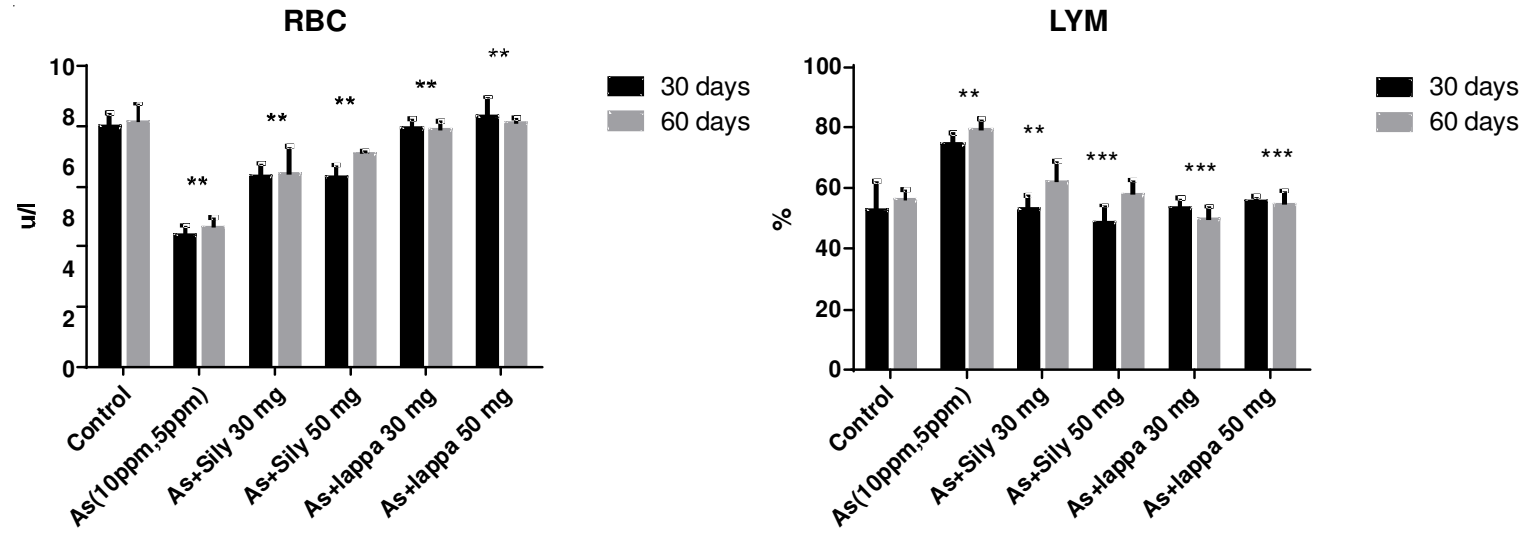

Fig. 6. Influence of various treatments on the RBC and Lymphocyte count. Data are expressed as mean \pm SD. The difference was found to be significant ( $p$ $<0.05)$ when the drug treated groups were compared with arsenic treated group

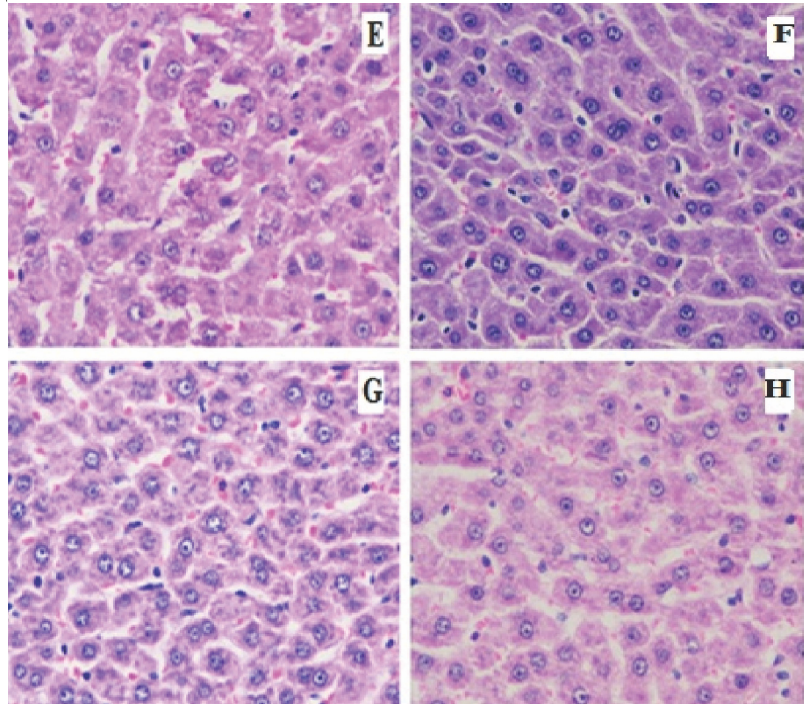

Fig. 7. Cross-section of liver tissues from the Silybum marianum administered animals after a treatment period of 30 days: A-Control group, B- Arsenic treated group, C-Arsenic + Silybum marianum low dose, D- Arsenic + Silybum marianum high dose
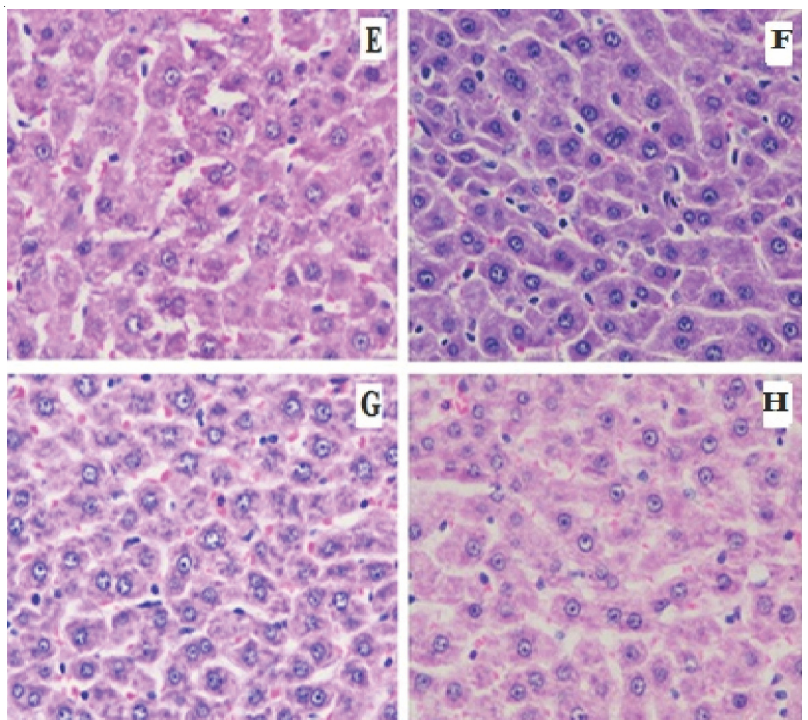

Fig. 8. Cross-section of liver tissues from the Arctium lappa administered animals after a treatment period of 60 days: E-Control group, FArsenic treated group, G-Arsenic + Arctium lappa low dose, HArsenic + Arctium lappa high dose the cells were discernible in all the groups. A similar observation was also made from the histopathological sections of the liver tissue from animals co-administered with Arctium lappa. Both for 30 and 60 days. No abnormal hepatic lesions or necrosis were reported. This due to the fact that before before arsenic trioxide could cause toxicity to the cells the co administration of the plant extracts has protected the cells from toxicity (Fig. 7 and 8).

\section{Conclusion}

From the results obtained from the biochemical, hematology and histopathology studies it may therefore be concluded that the co-administration of the plant extracts protects the hepatocytes from arsenic-induced changes and also signifies the fact that both Arctium lappa and Silybum marinum were found to exhibit the same level of potency indicating that Arctium lappa can be effective in ameliorating the toxicity of arsenic.

\section{ACKNOWLEDGEMENTS}

The authors are thankful to SASTRA University for poviding facilities to carry out the research work.

\section{REFERENCES}

1. M.L. Kile and D.C. Christiani, JAMA, 300, 845 (2008).

2. A. Manimaran, S.N. Sarkar and P. Sankar, Food Chem. Toxicol., 48, 605 (2010).

3. A. Navas-Acien, JAMA, 300, 814 (2008).

4. R. Chowdhury, A. Dutta, S.R. Chaudhuri, N. Sharma, A.K. Giri and K. Chaudhuri, Food Chem. Toxicol., 46, 740 (2008).

5. M.J. Mass, A. Tennant, B.C. Roop, W.R. Cullen, M. Styblo, D.J. Thomas and A.D. Kligerman, Chem. Res. Toxicol., 14, 355 (2001).

6. M. Gielen and E.R. Tiekink, Metallotherapeutic Drugs and Metal-Based Diagnostic Agents, Wiley, p. 298 (2005).

7. M.J. Gresser, J. Biol. Chem., 256, 5981 (1981).

8. T. Gebel, Toxicology, 144, 155 (2000).

9. L.M. Del Razo and B. Quintanilla-Vega, Toxicol. Appl. Pharmacol., 177, 132 (2001).

10. H. Greenlee, K. Abascal, E. Yarnell and E. Ladas, Integr. Cancer Ther., 6, 158 (2007).

11. J. Dai, R.S. Weinberg, S. Waxman and Y. Jing, Blood, 93, 268 (1999).

12. M. Vahter and G. Concha, Pharmacol. Toxicol., 89, 1 (2001).

13. K.T. Kitchin, Toxicol. Appl. Pharmacol., 172, 249 (2001).

14. F.S. Hogan, N.K. Krishnegowda, M. Mikhailova and M.S. Kahlenberg, J. Surg. Res., 143, 58 (2007).

15. J.J. Lah, W. Cui and K.-Q. Hu, World J. Gastroenterol., 13, 5299 (2007).

16. H.S. Kang, J.Y. Lee and C.J. Kim, J. Ethnopharmacol., 116, 305 (2008). 